\title{
Is it Time to Abandon 30-Day Mortality as a Quality Measure?
}

\author{
Rachel Hae-Soo Joung, MD, and Ryan P. Merkow, MD, MS, FACS $₫$ \\ Division of Surgical Oncology, Department of Surgery, Surgical Outcomes and Quality Improvement Center (SOQIC), \\ Feinberg School of Medicine, Northwestern University, Chicago, IL
}

Thirty-day operative mortality is one of the primary outcome measures used to assess and communicate risk, evaluate surgical safety, compare hospital quality, and provide a benchmark in public reporting and pay-for-performance initiatives. Fortunately, improvements in perioperative care have been largely successful at minimizing early treatment-related postoperative deaths. However, as perioperative care continues to advance with more life-sustaining therapies than historically available, many deaths occur beyond 30 days. Therefore, quality assessments based on 30-day events may actually underestimate the true risk. As such, there is a growing sentiment that 30-day mortality alone may not be the most appropriate measure of operative risk or surgical quality of care. Underestimating postoperative mortality can have major implications, such as shifting the risk profile towards favoring surgery in situations where there are alternative options and providing patients with inaccurate expectations regarding their operative risk. Thus, although there are many considerations and potential unintended consequences, there is increasing interest by the surgical community to extend the postoperative mortality timeframe from 30 to 90 days.

In this issue of Annals of Surgical Oncology, Resio et al. report the results of their study evaluating mortality events occurring within 90 days of surgery. ${ }^{1}$ Their goal was to characterize the circumstances surrounding postoperative deaths occurring between 31 and 90 days, which they term the "late" postoperative period. Using Surveillance,

(C) Society of Surgical Oncology 2021

First Received: 20 September 2020

Accepted: 4 October 2020;

Published Online: 3 January 2021

R. P. Merkow, MD, MS, FACS

e-mail: Ryan.Merkow@northwestern.edu
Epidemiology, and End Results (SEER)-Medicare data and the Connecticut Tumor Registry (CTR), patients older than 65 who underwent surgery for nonmetastatic primary cancers were evaluated. Their study confirms previously published findings; just as many patients died in the "late" postoperative period as they did in the "early" postoperative period. ${ }^{2-4}$ Additionally, most patients that died between 31 and 90 days initially recovered from surgery enough to be discharged from the hospital. They found that the most common cause of late mortality was attributed to malignancy itself in more than half of the cases. The majority of patients experiencing a late mortality event were readmitted at least once before death, and the primary diagnosis associated with this last readmission before death was infection.

Although this study provides important insights, probably more important are the many unanswered questions and issues it raises. First, due to data limitations and inherent challenges when determining the cause of death, the authors were unable to identify the actual underlying reason(s) for deterioration. More than half of the deaths were attributed to malignancy. The authors acknowledge that although "cancer" in a global sense may have led to these patients' demise (i.e., surgery would have never happened in the first place if the patients did not have cancer), given the relatively short time frame, malignancy itself was probably not the direct cause of death. National databases, including SEER-Medicare, do not provide enough granularity to identify the events and complications that lead to death.

Second, a major challenge of examining 90-day mortality is the need to delineate surgery-related complications from other factors. It is well established that patients are in a vulnerable state postoperatively, especially after extensive procedures. What is lesser known is the extent to which this vulnerable period impacts individual patients. In this study, the primary diagnosis associated with the last 
readmission before death most often was related to infection (26\%). More than over half were secondary to "sepsis." However, one must distinguish whether these infections are surgery-related (e.g., smoldering intra-abdominal infection related to an anastomotic leak) or infections secondary to an overall state of frailty and deconditioning. ${ }^{5}$ To add to the complexity is the fact that other adjuvant treatments, such as chemotherapy, are often already started during this timeframe, making it even more problematic to attribute risk to the surgery alone.

Third, from a hospital quality-assessment standpoint, 90- and 30-day mortality are unlikely to reflect the same underlying factors. Previous studies have shown that in addition to patient and hospital variables, such as age, comorbidities, and hospital volume that influence 30-day mortality, 90-day mortality also is influenced by distinct "cancer" factors, such as stage, location of the tumor, and receipt of neoadjuvant therapy. ${ }^{5}$ Hence, it is not surprising that studies have found that hospital performance rankings change when adjusting for 90-day mortality instead of 30-day mortality. ${ }^{5,6}$ Although we have been widely successful in driving down 30-day mortality for even the highest risk procedures, it remains unclear exactly how to impact these late mortality events.

A final consideration is that this study calls into question whether we should shift our focus on other measures of quality. Significant improvements in surgical technique, perioperative care, and postoperative management has significantly decreased the postoperative 30-day mortality rate across nearly all surgical procedures, even the most complex. Although mortality certainly has face validity with both the medical community and the public, and can easily be measured, it has low reliability due to the fact that it is now, fortunately, a relatively rare event. ${ }^{2,7}$ Moreover, when a 30-day death does occur, it often is difficult to pinpoint actionable quality improvement targets for individual procedures to prevent future events. In order for a quality measure to be useful, it must be reliable, measurable, and actionable. Thus, it may be more constructive to shift our focus on other measures of quality, such as morbidity, patient-centered metrics, appropriateness, efficiency, and equitable cancer care delivery. ${ }^{8}$
Although we are still left with many questions, there are some important takeaways from the study by Resio et al. ${ }^{1}$ We need to recognize that operative risk continues beyond 30 days after a major cancer operation, and 30-day mortality underestimates the true operative risk. To guide the next steps in improving surgical safety, we need more information and granular data with regards to the events surrounding deaths between 31 and 90 days, so that we can identify and decrease "preventable deaths." Finally, this study further motivates the need to define other more reliable, measurable, and meaningful quality metrics to continue to improve the safety of cancer surgery.

DISCLOSURES The authors declare no conflicts of interest.

\section{REFERENCES}

1. Resio BJ, Gonsalves L, Canavan M, et al. Where the other half dies: analysis of mortalities occurring more than 30 days after complex cancer surgery. Ann Surg Oncol. 2020. https://doi.org/10. 1245/s10434-020-09080-7.

2. Walters DM, McMurry TL, Isbell JM, Stukenborg GJ, Kozower BD. Understanding mortality as a quality indicator after esophagectomy. Ann Thorac Surg. 2014;98(2):506-11; discussion 511-2.

3. Visser BC, Keegan H, Martin M, Wren SM. Death after colectomy: it's later than we think. Arch Surg. 2009;144(11):1021-7.

4. Pezzi CM, Mallin K, Mendez AS, Greer Gay E, Putnam JB, Jr. Ninety-day mortality after resection for lung cancer is nearly double 30-day mortality. $J$ Thorac Cardiovasc Surg. 2014;148(5):2269-77.

5. In H, Palis BE, Merkow RP, et al. Doubling of 30-day mortality by 90 days after esophagectomy: a critical measure of outcomes for quality improvement. Ann Surg. 2016;263(2):286-91.

6. Adam MA, Turner MC, Sun Z, et al. The appropriateness of 30-day mortality as a quality metric in colorectal cancer surgery. Am J Surg. 2018;215(1):66-70.

7. Dimick JB, Welch HG, Birkmeyer JD. Surgical mortality as an indicator of hospital quality: the problem with small sample size. JAMA. 2004;292(7):847-51.

8. Merkow RP, Bilimoria KY, Ko CY. Surgical quality measurement: an evolving science. JAMA Surg. 2013;148(7):586-7.

Publisher's Note Springer Nature remains neutral with regard to jurisdictional claims in published maps and institutional affiliations. 